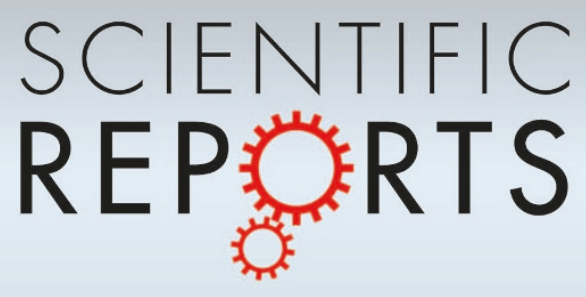

OPEN

SUBJECT AREAS:

SUPERCONDUCTING

PROPERTIES AND

MATERIALS

PHASE TRANSITIONS AND CRITICAL PHENOMENA

ELECTRONIC PROPERTIES AND MATERIALS

Received

31 March 2014

Accepted

2 June 2014

Published

11 July 2014

Correspondence and requests for materials should be addressed to

D.M. (Dragan.

Mihailovic@ijs.si)

\section{Separating pairing from quantum phase coherence dynamics above the superconducting transition by femtosecond spectroscopy}

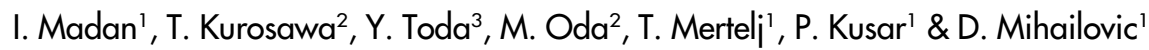

'Jozef Stefan Institute and International Postgraduate School, Jamova 39, S1-1000 Ljubljana, Slovenia, ${ }^{2}$ Department of Physics, Hokkaido University, Sapporo 060-0810, Japan, ${ }^{3}$ Department of Applied Physics, Hokkaido University, Sapporo 060-8628, Japan.

In classical superconductors an energy gap and phase coherence appear simultaneously with pairing at the transition to the superconducting state. In high-temperature superconductors, the possibility that pairing and phase coherence are distinct and independent processes has led to intense experimental search of their separate manifestations. Using femtosecond spectroscopy methods we now show that it is possible to clearly separate fluctuation dynamics of the superconducting pairing amplitude from the phase relaxation above the critical transition temperature. Empirically establishing a close correspondence between the superfluid density measured by $\mathrm{THz}$ spectroscopy and superconducting optical pump-probe response over a wide region of temperature, we find that in differently doped $\mathrm{Bi}_{2} \mathrm{Sr}_{2} \mathrm{CaCu}_{2} \mathrm{O}_{8+\delta}$ crystals the pairing gap amplitude monotonically extends well beyond $T_{\mathcal{c}}$ while the phase coherence shows a pronounced power-law divergence as $T \rightarrow T_{\mathcal{c}}$ thus showing that phase coherence and gap formation are distinct processes which occur on different timescales.

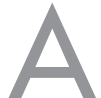

nomalous normal state behavior above the critical temperature appears to be a hallmark of unconventional superconductivity and is present in many different classes of materials. A pseudogap (PG) state has been suggested to be associated with a wide range of possible phenomena preceding the onset of macroscopic phase coherence at the superconducting (SC) critical transition temperature at $T_{c}:$ pre-formed pairs $^{1-9}$, a spin-gap $^{10}$, the formation of a Bose metal ${ }^{11}$, a Fermi or Bose glass, or a state composed of "dirty bosons" ${ }^{\text {"12-14 }}$, and more recently a charge-density-wave state ${ }^{15,16}$.

In addition to the PG response below the temperature designated as $T^{*}$, the response attributed to "superconducting fluctuations" above $T_{c}$ has been observed in a number of experiments ${ }^{17-27}$. The temperature region $T_{c}$ $<T<T_{\text {onset }}$ where such fluctuations are observable is significantly wider than in conventional superconductors, but smaller than $T^{*}$. The open and obvious question is whether the pseudogap, or the superconducting fluctuations can be attributed to pairing.

The problem in separating the response due to superconducting fluctuations from the PG is that so far, inevitably, one has had to make extrapolations, or assumptions about the response functions underlying temperature dependences and line shapes in transport ${ }^{17,18,28-30}$, magnetic susceptibility ${ }^{26,31}$, specific heat ${ }^{20,21}$ or photoemission (ARPES) ${ }^{22}$, which may at best introduce inaccuracies in the temperature scales, and at worst lead to erroneous conclusions. Alternatively one can suppress superconductivity by high magnetic fields up to $60 \mathrm{~T}^{32}$, although there exists a risk of inducing new states by such a high field ${ }^{33}$. Thus, so far it has not been possible to satisfactorily characterize superconducting fluctuations and discriminate between fluctuations of the amplitude $\delta \psi$ (related to the pairing gap) and phase $\delta \theta$ of the complex order parameter $\Psi=\psi e^{i \theta}$.

In pump-probe experiments three relaxation components shown in Fig. 1 a) are typically observed $\left.{ }^{34}: 1\right)$ the quasiparticle (QP) recombination in the SC state, 2) pseudogap state response below $T^{*}$ and 3) energy relaxation of hot electrons. The QP dynamics has been shown to be described very well by the Rothwarf-Taylor (R-T) model $^{35,36}$, and the response related to the presence of non-equilibrium QPs is thus unambiguous. Importantly, the presence of the QP response is directly related to the presence of a pairing gap for QP excitations.

Pump-probe experiments have already shown the coexistence of the pseudogap excitations with superconductivity below $T_{c}$ over the entire range of phase diagram ${ }^{37-41}$. However, little attention has been paid to the region of superconducting fluctuation. In this paper we present measurements by a 3-pulse technique which allows us to 

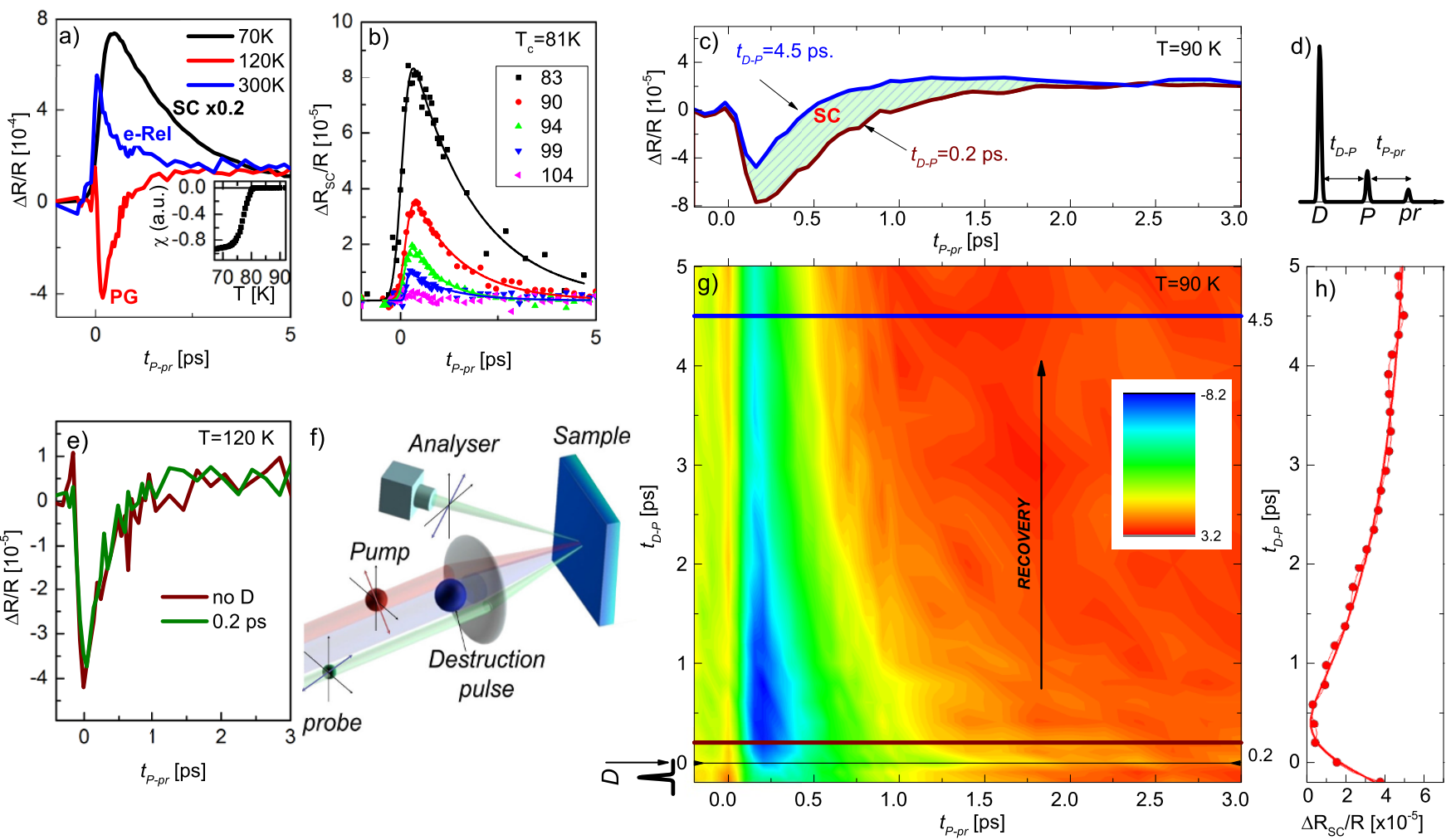

Figure $1 \mid$ Description of the 3 pulse method and pump-probe signals. Underdoped sample data: (a) Transient reflectivity signals observed at different temperatures in Bi-2212 - SC signal, the PG signal and energy relaxation of hot carriers (Inset: susceptibility curve shows $T_{c}=81 \mathrm{~K}$ for the underdoped sample). (b) The QP recombination at different $T$ above $T_{c}$. (c) Two typical 3-pulse pump-probe traces at $t_{D-P} 0.2$ and 4.5 ps show signal with suppressed and recovered superconducting component respectively. (d) Pulse sequence and delays notation in the 3-pulse experiment. (e) The pump-probe response at $120 \mathrm{~K}$ in the absence of the D-pulse and $0.2 \mathrm{ps}$ after the D pulse arrival. (The noise level is larger in comparison to c) due to a shorter signal accumulation time.) (f) A schematic representation of the 3-pulse experiment. Colors are schematic. Destruction (D) pulse destroys the superconductivity, Pump-probe sequence probes the recovery of the quasiparticle response. (g) A typical result of three pulse experiments at 90K shows suppression of the SC component after the $\mathrm{D}$ pulse arrival and gradual recovery with $t_{D-P}$. Readings along the blue and dark-red line are shown in Fig. $1 \mathrm{c}$ ). The color represents amplitude of the reflectivity change. The values of the color bars indicate $\Delta R / R \times 10^{-5}$. (h) Recovery of the superconducting component amplitude with $t_{D-P}$.

single out the response of superconducting gap fluctuations. The approach is based on selective destruction of the superconducting state by a femtosecond laser pulse ${ }^{42}$ which allows us to discriminate pseudogap excitations from superconducting fluctuation seen in transient reflectivity signals, thus avoiding the necessity of making extrapolations or assumptions in separating the different contributions. We then compare these data with a.c. conductivity $(\mathrm{THz})$ measurements from ${ }^{43}$ and establish proportionality of the amplitude of superconducting component in pump-probe experiment to the bare phase stiffness $\rho_{0}$, measured by $\mathrm{THz}$ experiments. This is directly proportional to the bare pair density $n_{s}^{23,43}$, which in turn coincides with the superfluid density when the latter is measured on a timescale on which changes of the order parameter due to either depairing or movement of the vortices can be neglected. Comparison of the critical behavior of the amplitude and phase correlation times above $T_{c}$ leads us to the conclusion that two quantities arise from different microscopic processes.

We perform measurements on under- (UD), near optimally-(OP) and over-(OD) doped $\mathrm{Bi}_{2} \mathrm{Sr}_{2} \mathrm{CaCu}_{2} \mathrm{O}_{8+\delta}$ (Bi2212) with $\mathrm{T}_{c} \mathrm{~s}$ of 81,85 and $80 \mathrm{~K}$ respectively. In the discussion we focus on the underdoped sample, and discuss comparisons with the optimally and overdoped samples, where applicable.

\section{Results}

Measurements of pairing amplitude above $T_{c}$. To separate the SC component from the $\mathrm{PG}$ component we use a 3 pulse technique described in Refs. 44-47, and schematically represented in Fig. 1f).
A pulse train of $800 \mathrm{~nm} 50 \mathrm{fs}$ pulses produced by a $250 \mathrm{kHz}$ regenerative amplifier is divided into three beams with variable delays. First a relatively strong "destruction" (D) pulse, with fluence just above the superconducting state photodestruction threshold, $F_{t h}^{S C}=13 \mu \mathrm{J} / \mathrm{cm}^{2}{ }^{48}$, destroys the superconducting condensate ${ }^{49}$. The ensuing recovery of the signal is measured by means of the 2 pulse Pump-probe (P-pr) response at a variable delay $t_{D-P}$ between $\mathrm{D}$ and $P$ pulses. There is no effect of the $D$ pulse on the $P G$ response within the noise level of $\sigma_{\Delta R / R} \lesssim 0.4 \times 10^{-5}$ indicated by measurements at $120 \mathrm{~K}$ shown in Fig. 1e). A typical result of the 3 pulse experiment is presented in Fig. 1g). In the absence of the $\mathrm{D}$ pulse the signal consists of a positive SC and a negative PG component. After the arrival of the $\mathrm{D}$ pulse we see a disappearance of the SC part and only the PG component is present (dark-red line in Fig. 1c)). With increasing $t_{D P}$ the superconducting response gradually re-emerges (blue line on Fig. 1c)). As most of the condensate in the probe volume is "destroyed" by the D pulse we can extract the superconducting component by subtracting the signal remanent after the destruction (measured $200 \mathrm{fs}$ after the D pulse) from the signal obtained in the absence of the D pulse. Such an extracted superconducting component is plotted in Fig. 1b). The signal is detectable up to $T_{\text {onset }}=104 \mathrm{~K}$, which is $0.28 T_{c}$ above $T_{c}$ but much lower than $T^{*} \approx 2.5 T_{c}$. In Fig. $1 \mathrm{~h}$ ) we show the recovery of the amplitude of the superconducting component $A_{s c}$ as a function of the time delay $t_{D-P}$ after the $\mathrm{D}$ pulse for $90 \mathrm{~K}$. The temperature dependence of the amplitude of the SC component measured by 3-pulse technique $A_{s c}^{3 p u l s e}$ is shown in Fig. 2b). 

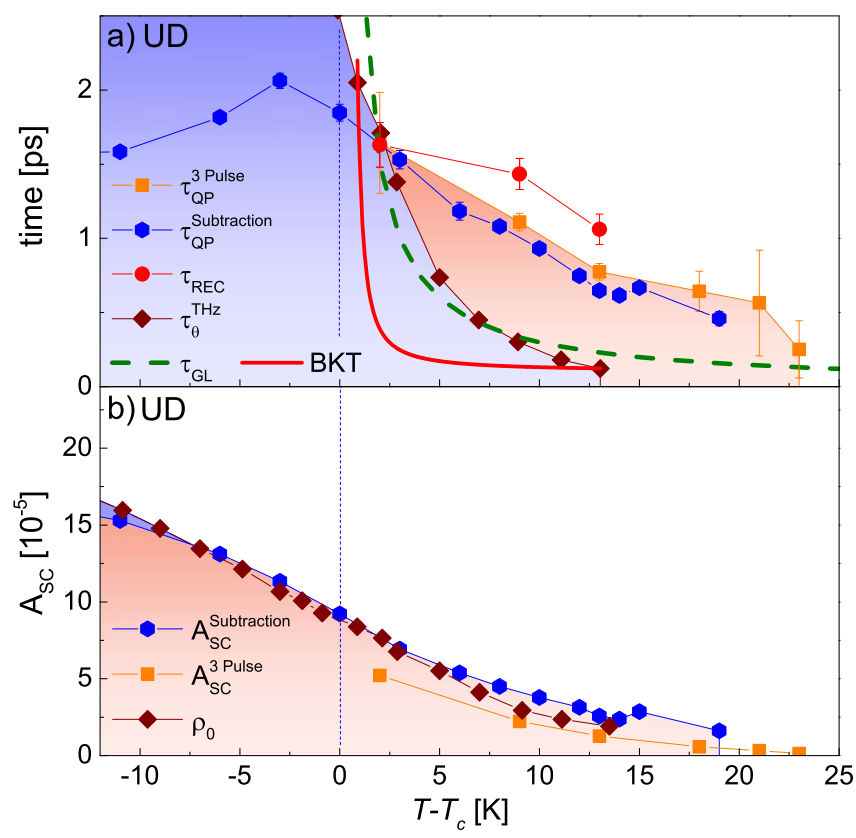

Figure 2 Comparison of pairing amplitude and phase coherence dynamics. (a) The recovery time of the optical superconducting signal $\left(\tau_{\text {rec }}\right)$, the QP recombination time $\tau_{Q P}^{3 P u l s e}$ measured by the three pulse

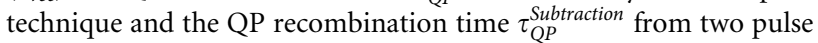
Pump-probe measurements obtained by subtraction of the PG signal. A fit to the data using a BKT model (eq. 3 of ref. 19) is shown by the solid red line. The dashed line shows the fluctuation lifetime $\tau_{G L}$ given by the TDGL theory. The phase correlation time $\tau_{\theta}^{T H z}$ obtained from the $\mathrm{THz}$ conductivity measurements ${ }^{43}$ is also shown for comparison. (b) The amplitude of the SC signal measured by the three pulse technique $\left(A_{S C}^{3 P u l s e}\right)$ and the two pulse measurements with the PG signal subtracted $\left(A_{S C}^{\text {Subtraction }}\right)$. The bare phase stiffness $\rho_{0}^{43}$ (normalized at $T_{c}$ ) shows remarkable agreement with the optical response.

For comparison, standard pump-probe measurements need to separate the SC relaxation from the PG relaxation by subtraction of the high temperature response extrapolated into the superconducting region. This approach suffers from the same uncertainties as other techniques such as conductivity, heat capacity, diamagnetism and ARPES. The actual $T$-dependence of the PG response can vary with doping, pump fluence and probe wavelength ${ }^{48,50}$. But, in Fig. 2b) we show that the subtraction procedure - with the use of a mode $^{48}$ - gives results in agreement with the direct 3 pulse measurements. The remaining discrepancies in the amplitude can be explained by an incomplete destruction of fluctuating superconducting state in the 3 pulse experiment and errors in the PG subtraction.

In Fig. 2a) we see that the QP relaxation time $\tau_{O P}^{3 P u l s e}$ obtained by fitting an exponential function to the data Fig. 1b) decreases rather gradually with increasing $T$ above $T_{c}$ and nearly coincide with the QP relaxation time obtained by the pseudogap subtraction procedure $\tau_{Q P}^{\text {Subtraction }}$. The recovery time $\tau_{\text {rec }}$ obtained from exponential fits to the recovery of the SC response above $T_{c}$ (Fig. $1 \mathrm{~h}$ ) shows a similar $T$ dependence. The experiments thus show that the recovery of the SC gap and the QP relaxation show very similar dynamics above $T_{c}$.

Comparison of optical and a.c. conductivity measurements. We now compare these data with $\mathrm{THz}$ measurements of the order parameter correlation time and bare phase stiffness ${ }^{19,43}$. The agreement between $\rho_{0}$ and $A_{s c}$ shown in Fig. 2b) is seen to be remarkably good over the entire range of measurements $0.8 T_{c}<$ $T<1.3 T_{c}$. This agreement is important because, taking into account $n_{s} \sim|\Psi|^{2}$, it validates the approximation that the pump-induced changes in the reflectivity or dielectric constant $\epsilon$ for small $n_{s}$ are related to the order parameter $\Psi$ as $\delta R \sim \delta \varepsilon \sim|\Psi|^{2}$.

In contrast to $A_{S C}$ and $\rho_{0}$, remarkable differences are seen in the temperature dependences of the characteristic lifetimes shown in Fig. 2a) obtained by optical techniques and $\mathrm{THz}$ conductivity measurements. The phase correlation time $\tau_{\theta}^{T H z}$ determined from the $\mathrm{THz}$ conductivity ${ }^{19}$ dies out very rapidly with increasing temperature, while the $T$-dependence of the amplitude relaxation $\left(\tau_{Q P}^{3 P u l s e}\right.$, $\tau_{Q P}^{\text {Subtraction }}$ or $\left.\tau_{R E C}\right)$ is much more gradual.

Measurements on an optimally doped sample (Fig. 3c, d))) show qualitatively the same results with $T_{\text {onset }} \sim 102 \mathrm{~K}$, which is $17 \mathrm{~K}$ above $T_{c}$, and slightly faster decrease of both amplitude and QP relaxation time with temperature. For the overdoped sample, $F_{t h}^{P G}$ becomes comparable to $F_{t h}^{S C}$, so the superconducting component cannot be significantly suppressed without affecting the pseudogap. Nevertheless the superconducting component is clearly observable in the 2-pulse response up to $T_{\text {onset }} \sim 93 \mathrm{~K}$, i.e. $13 \mathrm{~K}$ above $T_{c}$. Comparison of 2-pulse data for different doping levels is shown in Fig. 3 e)-g), showing qualitatively similar behavior of the SC amplitude above $T_{c}$.

\section{Discussion}

The co-existence and distinct dynamics of the PG and SC excitations above $T_{c}$ highlights the highly unconventional nature of these states. A possible explanation for the coexistence of the SC and PG excitations is that the SC and PG quasiparticles which are giving rise to the observed processes are associated with relaxation at different regions on the Fermi surface. Recent Raman and cellular dynamical mean-
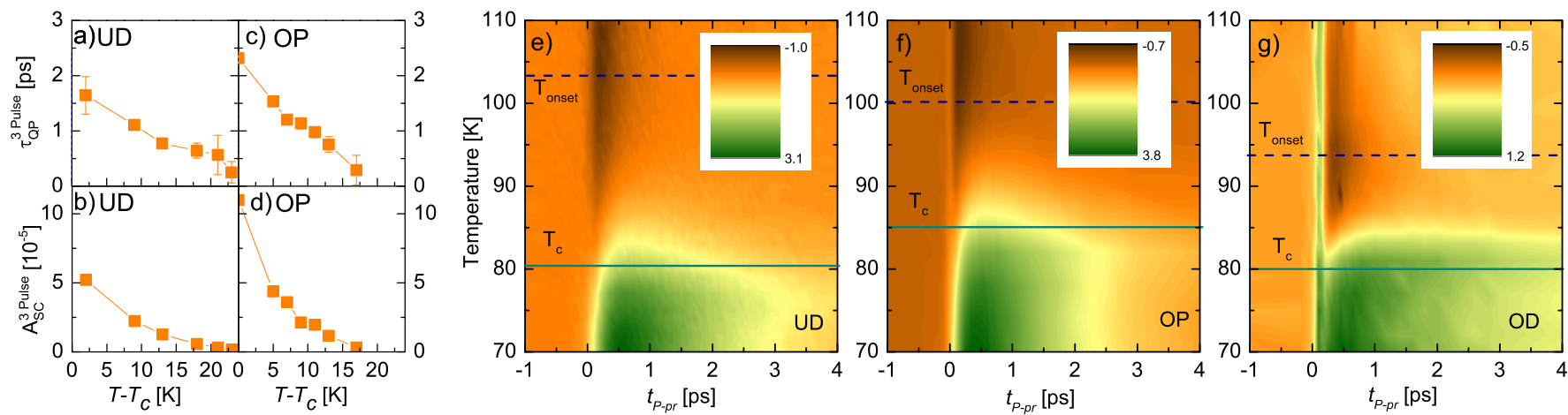

Figure $3 \mid$ Doping dependence. Comparison of the QP recombination time $\tau_{Q P}^{3 P u l s e}$ (a) and (c) and the amplitude of the SC signal (b) and (d) measured by the three pulse technique for under-(UD) and optimally(OP) doped samples, respectively. (e)-(g) T-dependence of 2-pulse response for under-(UD), optimally(OP) and overdoped(OD) samples. Blue dashed and cyan solid lines marks $T_{\text {onset }}$ and $T_{c}$ respectively. $T_{\text {onset }}$ shows gradual decrease with doping. The values of the color bars indicate $\Delta R / R \times 10^{-4}$. 
field studies ${ }^{51}$ have suggested that the PG may originate from the states inside the Mott gap, which are characterized by s-wave symmetry and very weak dispersion. Such a localized nature of the PG state excitations is consistent with previous assignments made on the basis of pump-probe experiments ${ }^{39,48}$. In contrast, the superconducting gap fluctuations have predominantly $d$-wave symmetry $^{52}$ and are more delocalized. This would explain the simultaneous presence of the SC fluctuations and PG components in pump-probe experiments.

Perhaps the most widely discussed model in the context of distinct pairing and phase coherence phenomena is the BerezinskiiKosterlitz-Thouless (BKT) transition ${ }^{53-55}$ by which decreasing temperature through $T_{\text {onset }}$ and approaching $T_{c}$ causes freely moving thermally activated vortices and anti-vortices to form pairs, thus allowing the condensate to acquire long range phase coherence in an infinite-order phase transition sharply at $T_{c}$. The bare pair density is finite to much higher temperatures (up to $T_{\text {onset }}$ ), where pairing is caused by a different mechanism, and the pseudogap is considered to be an unrelated phenomenon ${ }^{56-59}$. The effect is characterised in terms of a phase stiffness $\rho_{s}$, a quantity which characterizes the destruction of phase coherence by thermal fluctuations at a temperature $T_{c}=$ $T_{B K T}=\pi \rho_{s} / 8$. In cuprates $\rho_{s}$ is small due to reduced dimensionality and the low carrier density. Within this approach a.c. (THz) conductivity $^{19,43}$, heat capacity ${ }^{20,21}$, diamagnetism ${ }^{26,31}$, ARPES $^{22}$ and Nernst effect ${ }^{23-25,60}$ measurements were interpreted.

The BSCCO family is the most two-dimensional of the cuprate materials, so here the BKT mechanism for describing the order above $T_{c}$ would be expected to be most applicable ${ }^{43}$. However, the data in Fig. 2a) show that the drop in $\tau_{\theta}$ is not nearly as abrupt as the BKT model predicts. One possibility is that this broadening arises from chemical inhomogeneity of the sample, but the absence of a peak in the heat capacity at $T_{\text {onset }}{ }^{20}$ also appears to exclude the possibility of a pure BKT transition, and implies amplitude fluctuations might be present between $T_{c}$ and $T_{\text {onset }}$ as well ${ }^{21}$. Thus additional mechanisms beyond BKT may also be present which would broaden the phase coherence transition, such as interlayer phase fluctuations. In this case the observed $T$-dependence would reflect the interlayer decoherence $^{19,43}$.

In more traditional approaches using time-dependent GinzburgLandau (TDGL) theory ${ }^{61}$, thermal fluctuations are small for temperatures higher than $\sim 2 \mathrm{~K}$ above $T_{c}^{62}$, but can give an observable contribution to the conductivity in this temperature range $\mathrm{e}^{17,18,28-30}$. Relaxation within TDGL theory has a "longitudinal" relaxation time $\tau_{\Delta}$, which corresponds to the relaxation of the magnitude of the SC order parameter, and a "transverse" relaxation time $\tau_{\theta}$ which corresponds to the relaxation of its phase. They are related to each other in magnitude, but have the same temperature dependence near $T_{c}$, namely $\tau_{G L} \sim 1 /\left(T-T_{c}\right)^{63}$. Perhaps unexpectedly, the temperature dependence of $\tau_{\theta}^{T H z}$ nearly coincides with the behavior predicted for $\tau_{\theta}$ by time-dependent Ginzburg Landau theory for Gaussian fluctuations. Fig. 2a) suggests that phase coherence within this system is established in a narrow, $\sim 5 \mathrm{~K}$ temperature interval. However, the distinctly different critical behavior of the pair amplitude dynamics speaks in favor of unconventional models of superconductivity in which pairing and phase coherence occur independently, by different mechanisms. The implication is that the observed pairing amplitude which extends to more than $25 \mathrm{~K}$ above $T_{c}$ reflects the response of an inhomogeneous ensemble of gapped patches which are not mutually phase coherent. The weak temperature dependence of the amplitude cannot be described either by TDGL or BKT models.

Beyond the BKT vortex and TDGL scenario, other phase-locking scenarios, such as Bose-Einstein condensation of bipolarons $s^{2,3,64}$ and phase-coherence percolation ${ }^{4,5}$ may also be consistent with the observed behaviour. In both of these cases pairing and phase coherence are also distinct processes. The former comes from the condensation of pairs at $T_{c}$ as preformed pair kinetic energy is reduced, while percolation dynamics is associated with the time dynamics of Josephson tunneling between fluctuating pairs or superconducting patches. The percolation timescale $\tau_{J}$ is given by the Josephson energy $E_{J}=I_{c} \phi_{0} / 2 \pi$, where $I_{c}$ is the critical current and $\phi_{0}$ is the flux quantum. In cuprates, $\tau_{J}=\hbar / E_{J} \simeq 300 \mathrm{fs}$, which is compatible with the dynamics of phase shown in Fig. 2a).

A picture highlighted by Fig. 2 thus emerges in these materials where the relaxation of the phase $\theta$ is faster than relaxation of the amplitude $\psi$ of the complex order parameter $\Psi=\psi e^{i \theta}$, the dynamics of $\psi$ and $\theta$ being governed by microscopically different processes. It is worth remarking here that the opposite situation is found in charge density wave dynamics, where the phase relaxation is slow compared to the amplitude relaxation, and the dynamics can be described by TDGL equations for the amplitude $\psi$, neglecting phase relaxation $\theta^{45}$.

\section{Methods}

Samples. The samples used in this work were under-, near optimally- and overdoped Bi2212 with $T_{c} s$ of 81,85 and $80 \mathrm{~K}$ respectively. Samples were grown by the traveling solvent floating zone method. Critical temperatures were obtained from susceptibility measurements (e.g. inset in Fig. 1a) for the underdoped sample).

1. Alexandrov, A. S. \& Andreev, A. F. Gap and subgap tunnelling in cuprates Europhys. Lett. 54, 373-379 (2001).

2. Alexandrov, A. \& Ranninger, J. Bipolaronic superconductivity. Phys. Rev. B 24, 1164-1169 (1981).

3. Alexandrov, A. S. Theory of high-temperature superconductivity in doped polar insulators. Europhys. Lett. 95, 27004 (2011).

4. Mihailovic, D., Kabanov, V. V. \& Müller, K. A. The attainable superconducting $T_{c}$ in a model of phase coherence by percolating. Europhys. Lett. 57, 254-259 (2002)

5. Kresin, V.Z. \& Wolf, S. a. Inhomogeneous Superconducting State and Intrinsic $T_{c}$ : Near Room Temperature Superconductivity in the Cuprates. J. Supercond. Nov. Magn. 25, 175-180 (2011).

6. Ovchinnikov, Y. \& Kresin, V. Inhomogeneous superconductor in an ac field: Application to the pseudogap region. Phys. Rev. B 65, 214507 (2002).

7. Geshkenbein, V. B., Ioffe, L. B. \& Larkin, A. I. Superconductivity in a system with preformed pairs. Phys. Rev. B 55, 3173-3180 (1997).

8. Alexandrov, A. Bose-Einstein condensation of charged bosons in a magnetic field. Phys. Rev. B 48, 10571-10574 (1993).

9. Solov'ev, A. L. \& Dmitriev, V. M. Fluctuation conductivity and pseudogap in YBCO high-temperature superconductors (Review). Low Temp. Phys. 35, 169 (2009).

10. Emery, V. J. Spin-gap proximity effect mechanism of high-temperature superconductivity. Phys. Rev. B 56, 6120-6147 (1997).

11. Phillips, P. \& Dalidovich, D. The elusive Bose metal. Science 302, 243-7 (2003).

12. Das, D. \& Doniach, S. Existence of a Bose metal at $\mathrm{T}=0$. Phys. Rev. B 60, 1261-1275 (1999).

13. Das, D. \& Doniach, S. Bose metal: Gauge-field fluctuations and scaling for fieldtuned quantum phase transitions. Phys. Rev. B 64, 134511 (2001).

14. Vojta, M. Quantum phase transitions. Rep. Prog. Phys. 66, 2069-2110 (2003)

15. Torchinsky, D. H., Mahmood, F., Bollinger, A. T., Božović, I. \& Gedik, N. Fluctuating charge-density waves in a cuprate superconductor. Nat. Mater. 12, 387-91 (2013).

16. Sugai, S., Takayanagi, Y. \& Hayamizu, N. Phason and Amplitudon in the ChargeDensity-Wave Phase of One-Dimensional Charge Stripes in $\mathrm{La}_{2-x} \mathrm{Sr}_{x} \mathrm{CuO} \mathrm{O}_{4}$. Phys. Rev. Lett. 96, 137003 (2006)

17. Silva, E., Sarti, S., Fastampa, R. \& Giura, M. Excess conductivity of overdoped $\mathrm{Bi}_{2} \mathrm{Sr}_{2} \mathrm{CaCu}_{2} \mathrm{O}_{8}+x$ crystals well above $T_{c}$. Phys. Rev. B 64, 144508 (2001).

18. Truccato, M., Agostino, a., Rinaudo, G., Cagliero, S. \& Panetta, M. Possible dominance of the Maki-ThompsonThompson process in the fluctuation conductivity of Bi-2212 superconducting whiskers. J. Phys.: Condens. Matter 18, 8295-8312 (2006).

19. Orenstein, J., Corson, J., Oh, S. \& Eckstein, J. Superconducting fluctuations in $\mathrm{Bi}_{2} \mathrm{Sr}_{2} \mathrm{Ca}_{1-x} \mathrm{D} y_{x} \mathrm{Cu}_{2} \mathrm{O}_{8+\delta}$ as seen by terahertz spectroscopy. Ann. Phys. 15, 596-605 (2006)

20. Junod, A., Roulin, M., Revaz, B. \& Erb, A. Experimental survey of critical fluctuations in the specific heat of high-temperature superconductors. Physica B Condens. Matter 280, 214-219 (2000).

21. Tallon, J. L., Storey, J. G. \& Loram, J. W. Fluctuations and critical temperature reduction in cuprate superconductors. Phys. Rev. B 83, 092502 (2011).

22. Kondo, T. et al. Disentangling Cooper-pair formation above the transition temperature from the pseudogap state in the cuprates. Nat. Phys. 7, 21-25 (2010).

23. Wang, Y., Li, L. \& Ong, N. Nernst effect in high-Tc superconductors. Phys. Rev. B 73, 1-20 (2006).

24. Rullier-Albenque, F. et al. Nernst Effect and Disorder in the Normal State of High$T_{c}$ Cuprates. Phys. Rev. Lett. 96, 2-5 (2006).

25. Pourret, a. et al. Observation of the Nernst signal generated by fluctuating Cooper pairs. Nat. Phys. 2, 683-686 (2006). 
26. Li, L. et al. Diamagnetism and Cooper pairing above $T_{c}$ in cuprates. Phys. Rev. $B$ 81, 1-9 (2010).

27. Mihailović, D., Zgonik, M., Čopič, M. \& Hrovat, M. Quasiparticle excitations in the superconducting state observed in light scattering. Phys. Rev. B 36, 3997-3999 (1987)

28. Ghosh, A. K. \& Basu, A. N. Fluctuation-induced conductivity in quenched and

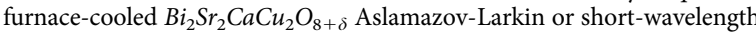
fluctuations. Phys. Rev. B 59, 11193-11196 (1999).

29. Balestrino, G., Crisan, A., Livanov, D., Manokhin, S. \& Milani, E. Fluctuation magnetoconductivity of BSCCO-2212 films in parallel magnetic field. Phys. C Supercond. 355, 135-139 (2001).

30. Bhatia, S. N. \& Dhard, C. Excess conductivity and pair-breaking effects in $Y$ $\mathrm{Ba}_{2} \mathrm{Co}_{3} \mathrm{O}_{7}$ and $\mathrm{Bi}_{2} \mathrm{Sr}_{2} \mathrm{Ca}_{1-x} \mathrm{Y}_{x} \mathrm{Cu}_{2} \mathrm{O}_{8+\delta}$ systems. Phys. Rev. B 49, 206-215 (1994).

31. Wang, Y. et al. Field-Enhanced Diamagnetism in the Pseudogap State of the Cuprate $\mathrm{Bi}_{2} \mathrm{Sr}_{2} \mathrm{CaCu}_{2} \mathrm{O}_{8+\delta}$ Superconductor in an Intense Magnetic Field. Phys. Rev. Lett. 95, 247002 (2005).

32. Rullier-Albenque, F., Alloul, H. \& Rikken, G. High-field studies of superconducting fluctuations in high- $T_{c}$ cuprates: Evidence for a small gap distinct from the large pseudogap. Phys. Rev. B 84, 014522 (2011).

33. Chen, Y. \& Ting, C. Magnetic-field-induced spin-density wave in hightemperature superconductors. Phys. Rev. B 65, 180513 (2002).

34. Gay, P., Smith, D. C., Stevens, C. J., Chen, C. \& Ryan, J. F. Femtosecond Dynamics of BSCCO-2212. Journal of Low Temperature Physics 117, 1025-1029 (1999).

35. Kaindl, R. A., Carnahan, M. A., Chemla, D. S., Oh, S. \& Eckstein, J. N. Dynamics of Cooper pair formation in $\mathrm{Bi}_{2} \mathrm{Sr}_{2} \mathrm{CaCu}_{2} \mathrm{O}_{8+\delta}$. Phys. Rev. B 72, 060510 (2005).

36. Kabanov, V. V., Demsar, J. \& Mihailovic, D. Kinetics of a Superconductor Excited with a Femtosecond Optical Pulse. Phys. Rev. Lett. 95, 147002 (2005).

37. Demsar, J., Podobnik, B., Kabanov, V. V., Wolf, T. \& Mihailovic, D. Superconducting Gap $\Delta_{c}$, the Pseudogap $\Delta_{p}$, and Pair Fluctuations above $T_{c}$ in Overdoped $\mathrm{Y}_{1-x} \mathrm{Ca}_{x} \mathrm{Ba}_{2} \mathrm{Cu}_{3} \mathrm{O}_{7-\delta}$ from Femtosecond Time-Domain Spectroscopy. Phys. Rev. Lett. 82, 4918-4921 (1999).

38. Kusar, P., Demsar, J., Mihailovic, D. \& Sugai, S. A systematic study of femtosecond quasiparticle relaxation processes in $\mathrm{La}_{2-x} \mathrm{Sr}_{x} \mathrm{CuO}$. Phys. Rev. B 72, 014544 (2005).

39. Kabanov, V. V., Demsar, J., Podobnik, B. \& Mihailovic, D. Quasiparticle relaxation dynamics in superconductors with different gap structures: Theory and experiments on $\mathrm{Y} \mathrm{Ba}_{2} \mathrm{Cu}_{3} \mathrm{O}_{7-\delta}$. Phys. Rev. B 59, 1497-1506 (1999).

40. Liu, Y. et al. Direct Observation of the Coexistence of the Pseudogap and Superconducting Quasiparticles in $\mathrm{Bi}_{2} \mathrm{Sr}_{2} \mathrm{CaCu}_{2} \mathrm{O}_{8+y}$ by Time-Resolved Optical Spectroscopy. Phys. Rev. Lett. 101, 1-4 (2008).

41. Nair, S. K. et al. Quasiparticle dynamics in overdoped $\mathrm{Bi}_{1.4} \mathrm{~Pb}_{0.7} \mathrm{Sr}_{1.9} \mathrm{CaCu}_{2} \mathrm{O}_{8+\delta}$ Coexistence of superconducting gap and pseudogap below $T_{c}$. Phys. Rev. B 82, 212503 (2010).

42. Kusar, P. et al. Controlled Vaporization of the Superconducting Condensate in Cuprate Superconductors by Femtosecond Photoexcitation. Phys. Rev. Lett. 101, 227001 (2008)

43. Corson, J., Mallozzi, R., Orenstein, J., Eckstein, J. \& Bozovic, I. Vanishing of phase coherence in underdoped $\mathrm{Bi}_{2} \mathrm{Sr}_{2} \mathrm{CaCu}_{2} \mathrm{O}_{8+\delta}$. Nature 398, 221-223 (1999).

44. Kusar, P. e. a. Coherent trajectory through the normal-to-superconducting transition reveals ultrafast vortex dynamics in a superconductor. arXiv:1207.2879v2 (2013).

45. Yusupov, R. et al. Coherent dynamics of macroscopic electronic order through a symmetry breaking transition. Nat. Phys. 6, 681-684 (2010).

46. Kusar, P. et al. Anharmonic order-parameter oscillations and lattice coupling in strongly driven $1 \mathrm{~T}-\mathrm{TaS}_{2}$ and $\mathrm{TbTe}_{3}$ charge-density-wave compounds: A multiple-pulse femtosecond laser spectroscopy study. Phys. Rev. B 83, 035104 (2011).

47. Mertelj, T. et al. Incoherent Topological Defect Recombination Dynamics in $\mathrm{TbTe}_{3}$. Phys. Rev. Lett. 110, 156401 (2013).

48. Toda, Y. et al. Quasiparticle relaxation dynamics in underdoped $\mathrm{Bi}_{2} \mathrm{Sr}_{2} \mathrm{CaCu_{2 }} \mathrm{O}_{8+\delta}$ by two-color pump-probe spectroscopy. Phys. Rev. B 84 174516 (2011)
49. Stojchevska, L. et al. Mechanisms of nonthermal destruction of the superconducting state and melting of the charge-density-wave state by femtosecond laser pulses. Phys. Rev. B 84, 180507 (2011).

50. Coslovich, G. et al. Competition Between the Pseudogap and Superconducting States of $\mathrm{Bi}_{2} \mathrm{Sr}_{2} \mathrm{Ca}_{0.92} \mathrm{Y}_{0.08} \mathrm{Cu}_{2} \mathrm{O}_{8+\delta}$ Single Crystals Revealed by Ultrafast Broadband Optical Reflectivity. Phys. Rev. Lett. 110, 107003 (2013).

51. Sakai, S. et al. Raman-Scattering Measurements and Theory of the EnergyMomentum Spectrum for Underdoped $\mathrm{Bi}_{2} \mathrm{Sr}_{2} \mathrm{CaCu}_{2} \mathrm{O}_{8+\delta}$ Superconductors: Evidence of an s-Wave Structure for the Pseudogap. Phys. Rev. Lett. 111, 107001 (2013).

52. Toda, Y. et al. Dynamics of broken symmetry nodal and anti-nodal excitations in $\mathrm{Bi}_{2} \mathrm{Sr}_{2} \mathrm{CaCu}_{2} \mathrm{O}_{8+\delta}$ probed by polarized femtosecond spectroscopy. arXiv:1311.4719 (2013)

53. Berezinskii, V. Destruction of Long-range Order in One-dimensional and Twodimensional Systems having a Continuous Symmetry Group I. Classical Systems. JETP 32, 493 (1971).

54. Berezinskii, V. L. Destruction of Long-range Order in One-dimensional and Two dimensional Systems Possessing a Continuous Symmetry Group. II. Quantum Systems. JETP 34, 610 (1972).

55. Kosterlitz, J. M. \& Thouless, D. J. Ordering, metastability and phase transitions in two-dimensional systems. J. Phys. C 6, 1181-1203 (1973).

56. Emery, V. J. \& Kivelson, S. A. Importance of phase fluctuations in superconductors with small superfluid density. Nature 374, 434-437 (1995).

57. Emery, V., Kivelson, S. \& Zachar, O. Pairing and phase coherence in high temperature superconductors. Phys. C Supercond. 282-287, 174-177 (1997).

58. Emery, V. \& Kivelson, S. Crossovers and phase coherence in cuprate superconductors. J. Phys. Chem. Solids 59, 1705-1710 (1998).

59. Fisher, D., Fisher, M. \& Huse, D. Thermal fluctuations, quenched disorder, phase transitions, and transport in type-II superconductors. Phys. Rev. B 43, 130-159 (1991).

60. Mukerjee, S. \& Huse, D. Nernst effect in the vortex-liquid regime of a type-II superconductor. Phys. Rev. B 70, 014506 (2004).

61. Larkin, A. \& Varlamov, A. Theory of Fluctuations in Superconductors (Oxford University Press, 2005).

62. van der Beek, C., Colson, S., Indenbom, M. \& Konczykowski, M. Supercooling of the Disordered Vortex Lattice in $\mathrm{Bi}_{2} \mathrm{Sr}_{2} \mathrm{CaCu}_{2} \mathrm{O}_{8+\delta}$. Phys. Rev. Lett. 84, 4196-4199 (2000).

63. Schuller, I. \& Gray, K. E. Experimental Observation of the Relaxation Time of the Order Parameter in Superconductors. Phys. Rev. Lett. 36, 429-432 (1976).

64. Alexandrov, A. S. High-temperature superconductivity: the explanation. Phys. Scr. 83, 038301 (2011).

\section{Author contributions}

T.K., Y.T. and M.O. has grown the samples and done magnetic characterisation, I.M. did optical measurements. I.M. and P.K. performed data analysis. I.M., Y.T., T.M. and D.M. interpreted the data. I.M and D.M. wrote the manuscript.

\section{Additional information}

Competing financial interests: The authors declare no competing financial interests.

How to cite this article: Madan, I. et al. Separating pairing from quantum phase coherence dynamics above the superconducting transition by femtosecond spectroscopy. Sci. Rep. 4, 5656; DOI:10.1038/srep05656 (2014).

This work is licensed under a Creative Commons Attribution-NonCommercialNoDerivs 4.0 International License. The images or other third party material in this article are included in the article's Creative Commons license, unless indicated otherwise in the credit line; if the material is not included under the Creative Commons license, users will need to obtain permission from the license holder in order to reproduce the material. To view a copy of this license, visit http:// creativecommons.org/licenses/by-nc-nd/4.0/ 